\title{
Protection of mouse pancreatic islet function by co-culture with hypoxia pre-treated mesenchymal stromal cells
}

\author{
CHENG XIANG and QIU-PING XIE \\ Department of Surgery, The Second Affiliated Hospital of Zhejiang University, Hangzhou, Zhejiang 310009, P.R. China
}

Received June 7, 2017; Accepted April 19, 2018

DOI: $10.3892 / \mathrm{mmr} .2018 .9235$

\begin{abstract}
Ectogenic pancreatic islet transplantation has long been discussed as having the potential to reverse diabetes. The aim of the present study was to evaluate the therapeutic efficacy of co-transplantation with hypoxia pretreated mesenchymal stem cells (MSCs) and islets in a diabetic mouse model. MSCs were isolated from femoral and tibial bone marrow aspirates from female BALB/c donor mice. MSC proliferation rates and the expression levels of vascular endothelial growth factor A (VEGFA), interleukin (IL)-6, monocyte chemoattractant protein (MCP)-1 and matrix metalloproteinase (MMP)-9 were measured in hypoxic conditions. Subsequently, a streptozotocin-induced diabetic model was established in BALB/c mice. Glucose tolerance and diabetes reversal rate following co-transplantation of hypoxia pre-cultured MSCs and islets were demonstrated at different conditions during transplantation. The present study results demonstrated that MSCs increased their proliferation rate and the secretion of growth-related cytokines, including VEGFA, IL-6, MCP-1 and MMP-9 in a hypoxic environment. In the diabetes animal model, fewer islets $(\sim 250)$ were required to reverse the impaired glucose tolerance condition in Islets + Hypoxia cultured MSCs transplant group compared with the Islets-only group ( 400 islets) and the Islets + Normal cultured MSCs group ( 300 islets). Hypoxia-cultured MSC co-transplantation accelerated glycemic utilization following glucose intake. In subjects with hyperglycemia control for islet only transplantation group, MSCs pre-cultured in hypoxic condition prior to co-transplantation may potentially improve islet tissue regeneration.
\end{abstract}

Correspondence to: Dr Qiu-Ping Xie, Department of Surgery, The Second Affiliated Hospital of Zhejiang University, 88 Jie Fang Road, Shangcheng, Hangzhou, Zhejiang 310009, P.R. China

E-mail: yushengxqp@zju.edu.cn

Abbreviations: BMDC, bone marrow derived cell; CM, conditioned medium; IL, interleukin; MCP, monocyte chemoattractant protein; MSCs, mesenchymal stem cells; RT-qPCR, reverse transcription-quantitative polymerase chain reaction; STZ, streptozotocin; VEGF, vascular endothelial growth factor

Key words: mesenchymal stem cells, islet transplantation, diabetes, hypoxia, glycemia

\section{Introduction}

Diabetes mellitus is the most prevalent endocrine and metabolic disease worldwide and is characterized by hyperglycemia caused by either impaired insulin secretion in the pancreas or by insulin resistance (1). Many of the patients with diabetes that fail to control their blood glucose levels may develop hypertension and cardiovascular diseases, which may contribute to the high morbidity and mortality rates in adults. In addition, $<50 \%$ insulin dependent patients achieve the recommended HB A1c (4-6\%) and exogenous insulin is unable to provide the same level of glycemic control as insulin secreted by the pancreas $(2,3)$. To date, transplantation of intact pancreas or isolated pancreatic islets appears to be a promising treatment for patients with type 1 diabetes. For example, it was previously reported that islet transplantation significantly decreased the mean amplitude of glycemic fluctuations in a study involving seven patients with type 1 diabetes (4). However, another study reported that grafted islet function progressively decreased following surgery and $44 \%$ of patients become insulin independent after 3 years (5), which indicated that diabetes may be successfully reversed in only a portion of patients (6).

Mesenchymal stem cells (MSCs) are a subpopulation of multipotent cells that were first identified in myeloid tissue (7). Several studies have demonstrated that co-transplantation with MSCs may improve islet graft survival rate through various mechanisms, such as immune modulation and enhancement of revascularization by cytokine secretion, including vascular endothelial growth factor (VEGF), hepatocyte growth factor, platelet-derived growth factor and matrix metalloproteinases (MMPs) (8-15). Nevertheless, previous studies have focused only on pancreatic islet allografts as an experimental model in normoxic microenvironmental conditions $(8,16,17)$. Conversely, in native bone marrow tissue, low oxygen tension $(2 \%)$ is considered an integral component of human MSC proliferation (18). Furthermore, a hypoxic environment was reported to have a close association with the biological characteristics of MSCs. One study reported that culturing MSCs under hypoxic conditions prior to transplantation may improve their tissue regenerative ability (19). Hypoxia-stimulated MSCs may secret a higher level of anti-apoptotic and antigenic related factors, including interleukin (IL)-6, VEGF and monocyte chemoattractant protein (MCP)-1. As a result, the hypoxia-cultured MSC conditioned medium (hMSC-CM) may possess the ability to suppress apoptosis and enhance 
angiogenesis of vascular endothelial cells (20). Therefore, pre-treating MSCs in a hypoxic microenvironment may benefit pancreatic islet transplantation graft survival rate.

The present study examined the proliferation rates and the expression levels of several growth factors in hypoxia-cultured MSCs. An in vivo co-transplantation model was established using hMSCs and pancreatic islet allografts in streptozotocin (STZ)-induced diabetic mice to evaluation the transplantation effect. The present study also investigated intravenous glucose tolerance and minimal islet number following islet transplantation with hMSCs or MSCs, providing information about the cross-protective effects of hMSCs with islet transplantation.

\section{Materials and methods}

Bone marrow-derived cell (BMDC) and MSC isolation, culture, adipose induction and oil red-O staining. BMDCs, which contained MSCs, were isolated from mouse bone marrow aspirates by adhesion to tissue culture-coated plates in complete medium as previously described (21). Briefly, BMDCs were isolated from bone marrow aspirates flushed from the femur and tibia of 20 female BALB/c donor mice (age, 9-10 weeks, $21 \pm 1 \mathrm{~g}$, Shanghai SLAC Laboratory Animal Co., Ltd., Shanghai, China). Mice were kept at $18-29^{\circ} \mathrm{C}$, with 40-70\% humidity, 12-h light/dark cycle and free access to food and water. Extracted BMDCs were cultured for 3-4 weeks using MSC medium composed of Iscove's modified Dulbecco's medium (Gibco; Thermo Fisher Scientific, Inc., Waltham, MA, USA) supplemented with $10 \%$ (v/v) fetal calf serum (Gibco; Thermo Fisher Scientific, Inc.), 10\% (v/v) equine serum (HyClone; GE Healthcare Life Sciences, Logan, UT, USA), $1 \%(\mathrm{v} / \mathrm{v})$ L-glutamine and $1 \%(\mathrm{v} / \mathrm{v})$ antibiotic-antimycotic liquid (Gibco; Thermo Fisher Scientific, Inc., Waltham, MA, USA). The culture medium was replaced every 3-4 days. At $60-80 \%$ confluence, cells were treated with $0.25 \%(\mathrm{w} / \mathrm{v})$ trypsin containing $1 \mathrm{mmol} / \mathrm{l}$ EDTA (Gibco; Thermo Fisher Scientific, Inc., Waltham, MA, USA) and passaged until passage 7 at a cell density of 2,500 cells $/ \mathrm{cm}^{2}$. Cultures were maintained at $37^{\circ} \mathrm{C}$ and $5 \% \mathrm{CO}_{2}$ with $2 \%$ (hypoxia) or $21 \%$ (normoxia) $\mathrm{O}_{2}$ concentration. MSC adipose induction was performed using an MSC adipogenic differentiation medium kit, (cat. no. GUXMX-90031; Cyagen Biosciences Inc., Santa Clara, CA, USA) following the manufacturer's protocols. Following the differentiation of the cells, the MSC adipogenic differentiation medium was removed from the wells which were rinsed with 1X phosphate-buffered saline (PBS). The cells were fixed with $2 \mathrm{ml}$ of $4 \%$ formaldehyde solution for $30 \mathrm{~min}$ at $37^{\circ} \mathrm{C}$. Oil Red O (Sigma-Aldrich; Merck KGaA, Darmstadt, Germany) working solution was prepared with saturated Oil Red O liquid ( $0.5 \mathrm{~g}$ Oil Red O dissolved in $100 \mathrm{ml}$ isopropanol) and distilled water with a 3:2 ratio. Staining was conducted with freshly prepared Oil Red O for 15 mins at room temperature, and rinsed with $60 \%$ isopropanol prior to inverted microscope analysis.

Cell viability assay. Cell viability was measured by MTT (Sigma-Aldrich; Merck KGaA) dye absorbance, according to the manufacturer's protocol (Boehringer Mannheim; Roche Diagnostics GmbH, Mannheim, Germany). Cells were incubated until desired density (60-80\% confluence). Formazan crystals were dissolved with DMSO and cell viability was determined spectrophotometrically at a wavelength of $570 \mathrm{~nm}$. Each experiment comprised five identical wells and was repeated three times.

Hypoxic culture and MSC-CM collection. An hypoxic environment was generated and maintained using a hypoxia chamber (Stemcell Technologies, Inc., Vancouver, BC, Canada), according to the manufacturer's protocol. Briefly, cultures were enclosed in the chamber that was flushed with a mixture of gasses $\left(93 \% \mathrm{~N}_{2}\right.$ and $5 \% \mathrm{CO}_{2}$ ) for $3 \mathrm{~min}$. Following the flushing period, the chamber was closed to prevent the flow of exogenous normoxic air into the chamber. The final level of hypoxia was $2 \%$ as specified by the manufacturer. Serum-free IMDM medium was used during CM production. Prior to culture, MSCs were counted and $3 \times 10^{4}$ cells were plated in the different groups. Following $24 \mathrm{~h}$ culture at $37^{\circ} \mathrm{C}$, the $\mathrm{CM}$ was harvested and used in subsequent experiments; the CM was applied at a 30:70 ratio with normal medium for islets culture.

Enzyme linked immunosorbent assay (ELISA). Cells was cultured until $70 \%$ confluence before CM harvest. Competitive ELISA was performed as previously described (22). Briefly, 96-well plate Elisa kits were coated with monoclonal mouse antibodies against VEGF (cat. no. VAL608), IL-6 (cat. no. VAL604), MCP-1 (cat. no. MJE00B) and MMP-9 (cat no. MMPT90) (R\&D Systems, R\&D Systems Inc, Minneapolis, USA). MSC-CM was harvested in 24, 48, 72 and $96 \mathrm{~h}$ following treatment. MSC-CM and standard test fluid incubations were performed in duplicate, including serial dilutions of the standard $(0.005-500 \mathrm{pmol} / \mathrm{ml})$ or MSCs CM for $4 \mathrm{~h}$ at room temperature. Biotinylated secondary antibodies (B-2763; 1:2,000; Invitrogen; Thermo Fisher Scientific, Inc.) were incubated at $37^{\circ} \mathrm{C}$ for $30 \mathrm{~min}$ to reveal bound antibodies. The reaction was stopped with $\mathrm{HCl}(1 \mathrm{~mol} / \mathrm{l})$ and the optical density was measured at $450 \mathrm{~nm}$ using a multilabel plate reader (Hidex Oy, Turku, Finland).

Diabetes induction and co-transplantation of MSCs and islets. A total of 80 Recipient female BALB/c mice (age, 8-9 weeks age, $21 \pm 1 \mathrm{~g}$ ) were included, and each group contained 10 mice. Mice were kept at $18-29^{\circ} \mathrm{C}$, with $40-70 \%$ humidity, 12-h light/dark cycle and free access to food and water. Mice were injected with STZ $(225 \mathrm{mg} / \mathrm{kg}$ ) ( Sigma-Aldrich; Merck KGaA; St. Louis, Missouri; USA) to induce diabetes 5-7 days prior to islet transplantation and were considered fit for transplantation if the non-fasting blood glucose concentration was $>11.1 \mathrm{mmol} / \mathrm{l}$ for 2 consecutive days. Diabetic female BALB/c mice were anaesthetized $(100 \mathrm{mg} / \mathrm{kg}$ ketamine and $10 \mathrm{mg} / \mathrm{kg}$ xylazine) and a marginal mass of 250-400 islet equivalents with or without $2.5 \times 10^{5} \mathrm{MSCs}$ was centrifuged (100 x g for $2 \mathrm{~min}$ at $37^{\circ} \mathrm{C}$ ) and transplanted under the kidney capsule.

Flow cytometry. MSC surface antigens were analyzed by flow cytometry. MSCs were trypsinized, resuspended $\left(1 \times 10^{6} / \mathrm{ml}\right)$ in serum-free MSC medium and incubated at $4^{\circ} \mathrm{C}$ for $20 \mathrm{~min}$ with fluorescein isothiocyanate (FITC)-conjugated anti-cluster of differentiation CD34 (555822), anti-CD45 (550539), anti-CD29 (553715), and anti-CD90 (553016; BD Biosciences, Franklin Lakes, NJ, USA). Cells were further incubated for $10 \mathrm{~min}$ at 
room temperature with 7-aminoactinomycin D (7-AAD) and subsequently washed and analyzed using a flow cytometer (BD Biosciences). The islet apoptosis rate was determined by fluorescence-activated cell sorting (FACS) flow cytometry and an Annexin V staining kit (11858777001; Roche Diagnostics, Basel, Switzerland). After $48 \mathrm{~h}$ incubation, the supernatant (floating apoptotic cells) was collected and the adherent cells from each well trypsinized. The collected cells were washed twice with PBS and centrifuged at $670 \mathrm{x}$ g for $5 \mathrm{~min}$ at room temperature. Each pellet was re-suspended in PBS (400 $\mu \mathrm{l})$. The signal intensity was acquired by a FACS Calibur (BD Biosciences) flow cytometer and analyzed with affiliated software (BD CellQuest Pro Software version 5.1, BD Biosciences). Propidium iodide (PI) negative and Annexin V negative were considered healthy cells, PI negative and Annexin V positive were considered apoptotic, and cells positive to both PI and Annexin V considered necrotic.

In vitro insulin and release quantitation. Cells were initially incubated for $3 \mathrm{~h}$ in glucose-free IMDM medium. This was followed by incubation for $1 \mathrm{~h}$ in medium containing 2.7, 11.1 or $27 \mathrm{mM}$ glucose. The supernatant was collected at the end of each incubation. The collected samples were frozen at $-70^{\circ} \mathrm{C}$ until assayed using an insulin ELISA kit (EIA-3439; DRG Instruments GmbH, Marburg, Germany).

Reverse transcription-quantitative polymerase chain reaction $(R T-q P C R)$. Total RNA was prepared by using TRIzol reagent (Life Technologies; Thermo Fisher Scientific, Inc.) from cells at $\sim 80 \%$ confluence. RNA purity and quantification assessment was performed with an ultraviolet spectrophotometer and the 260OD/280OD absorbance ratio was calculated (ratios of 1.8-2.0 indicated good purity and quantification. For cDNA synthesis, random sequence primers were used to prime the RT reactions, and synthesis was carried out by SuperScript III Reverse Transcriptase (Invitrogen; Thermo Fisher Scientific, Inc. USA). PCR cycling conditions were: Enzyme activation, $95^{\circ} \mathrm{C}$ for $30 \mathrm{sec}$, one cycle; denaturation, $95^{\circ} \mathrm{C}$ for $5 \mathrm{sec}, 40$ cycles; annealing, $55-60^{\circ} \mathrm{C}$ for $20 \mathrm{sec}, 40$ cycles. Standard curves were represented for each target gene and for the endogenous reference (GAPDH) in each sample. The quantification of the samples was performed by the LightCycler 480 Real-Time PCR System (Roche Diagnostics). Primer sequences were designed using Primer premier version 6.0 software (Premier Biosoft International, Palo Alto, CA, USA). Ideal primer sequences are listed below (Table I). The $2^{-\Delta \Delta C q}$ method was used to quantify gene expression (23). GAPDH was amplified in separate wells as reference gene. Each experiment comprised five identical wells and was repeated three times.

Apoptosis detection by fluorescein diacetate (FDA)-propidium iodide $(P I)$ viability staining. Islet preparations were assessed for islet cell viability using cell membrane exclusion dyes FDA and PI both from (Sigma-Aldrich; Merck KGaA; St. Louis, Missouri; USA) staining as previously described (24). The samples were examined using a fluorescent microscope, visualized and photographed. Apoptotic cells were stained red and viable cells were stained green. A total of 5 non-overlapping fields were imaged and the red stained cells were counted out of the total number of cells to determine the apoptosis rate.
mRNA microarray assay. Mice serum was collected and separated from blood following sacrifice. Total serum RNA was extracted using TRIzol reagent (Life Technologies; Thermo Fisher Scientific, Inc.). RNA purity and quantification was assessed by ultraviolet spectrophotometer scanning, $2600 D / 2800 D$ suction photometric ratio was calculated as described above using the Agilent Bioanalyzer 2100 (Agilent Technologies, Inc., Santa Clara, CA, USA). The samples were cleaned and hybridized according to the Agilent 2100 expert software B.02.07 (Agilent Technologies, Inc.). Original data were obtained using the mRNA Microarray Chip Mouse Gene 2.0 ST Array and the Affymetrix Gene Chip Command Console version 4.0 and analyzed with the Expression Console software version 1.1 (Affymetrix; Thermo Fisher Scientific, Inc. California, USA).

Ethical approval. All procedures performed using animals were in accordance with the ethical standards of the institution and the study was approved by the ethics committee of The Second Affiliated Hospital of Zhejiang University (Hangzhou, China).

Statistical analysis. All experimental values were presented as the mean \pm standard deviation, and the differences of mean values were statistically evaluated by one-way analysis of variance followed by Student-Newman-Keuls post hoc test using SPSS software version 18 (SPSS, Inc., Chicago, IL, USA). $\mathrm{P}<0.05$ was considered to indicate a statistically significant difference.

\section{Results}

BMDC and MSC characterization. By the third passage, a homogeneous population of fibroblast-like cells was obtained (Fig. 1A). Cells from passages 3-7 were used throughout the present study. Characterization by flow cytometry confirmed the absence of cells expressing the hematopoietic markers CD34 (0.53 $\pm 0.12 \%)$ and CD45 (1.03 $\pm 0.14 \%)$. BMDCs that expressed CD29 (36.32 $\pm 1.42 \%)$ and CD90 $(40.68 \pm 2.31 \%$; Fig. 1B), which is a typical feature of cell group with large proportion of mesenchyme cells and certain fibroblast cells $(25,26)$, were detected to identify MSCs.

Hypoxia treatment promotes MSC proliferation and induces growth-related cytokine expression. To investigate the effects of hypoxia on MSCs, MSCs were plated in 96-well plates with $15 \%$ fetal bovine serum and the growth rate was measured every day. hMSCs were able to form a greater number and larger-sized adipose globules compared with those formed by normoxia-cultured MSCs (nMSCs), as determined by adipose induction experiments (Fig. 2A). The results also revealed a higher proliferation rate in hMSCs compared with nMSCs (Fig. 2B). MSCs secrete a number of soluble factors that are involved in MSC growth, angiogenesis and autoimmune status (27). In the ELISA experiment, VEGFA, IL-6, MCP-1 and MMP-9 mRNA expression levels were measured in nMSCs and hMSCs. The average mRNA expression levels of VEGFA (1.73-fold increase), IL-6 (1.31-fold increase), MCP-1 (2.13-fold increase) and MMP-9 (1.63-fold increase) were significantly higher in hMSCs compared with the nMSC 
Table I. Primer sequences.

Annealing

Gene $\quad$ Sequence (5'-3')
Product size (bp)

\begin{tabular}{|c|c|c|c|}
\hline \multirow[t]{2}{*}{ VEGF } & F: 5-ATCTTCAAGCCGTCCTGTGTGC-3 & \multirow[t]{2}{*}{120} & \multirow[t]{2}{*}{60} \\
\hline & R: 5-TTGGCTTGTCACATTTTTCTGG-3 & & \\
\hline \multirow[t]{2}{*}{ IL-6 } & F: 5-ATGAAGTTCCTCTCTGCAAGAGAC-3 & \multirow[t]{2}{*}{108} & \multirow[t]{2}{*}{60} \\
\hline & R: 5-CACTAGGTTTGCCGAGTAGATCTC-3 & & \\
\hline \multirow[t]{2}{*}{ MCP-1 } & F: 5-TGTCTGGACCCCATTCCTTC-3 & \multirow[t]{2}{*}{140} & \multirow[t]{2}{*}{55} \\
\hline & R: 5-ACCAGCAAGATGATCCCAAT-3 & & \\
\hline \multirow[t]{2}{*}{ MMP-9 } & F: 5-CCTGGAACTCACACGACATCTTC-3 & \multirow[t]{2}{*}{132} & \multirow[t]{2}{*}{55} \\
\hline & R: 5-TGGAAACTCACACGCCAGAA-3 & & \\
\hline \multirow[t]{2}{*}{ GAPDH } & F: 5-GGAGAGAACCTGGTCCTCAG-3 & \multirow[t]{2}{*}{300} & \multirow[t]{2}{*}{55} \\
\hline & R: 5-ACCCAGAAGACTGTGGATGG-3 & & \\
\hline
\end{tabular}

F, forward; IL, interleukin; R, reverse; MCP-1, monocyte chemoattractant protein; MMP, matrix metalloproteinase; VEGF, vascular endothelial growth factor.

A
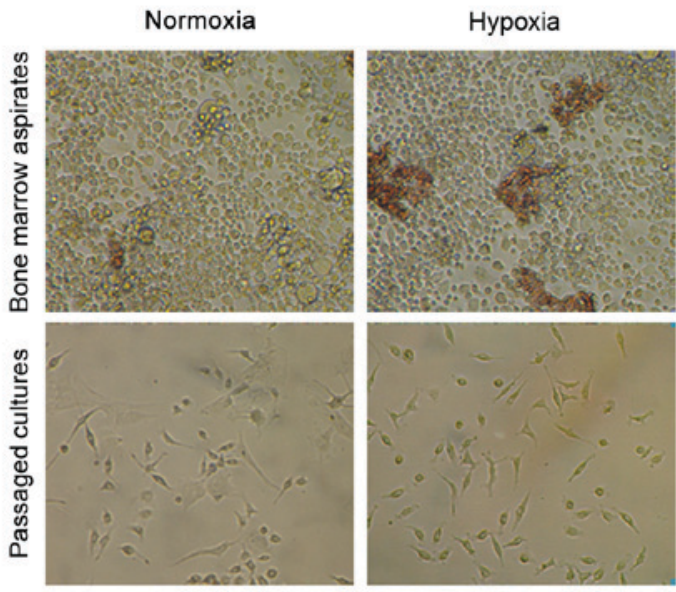

B
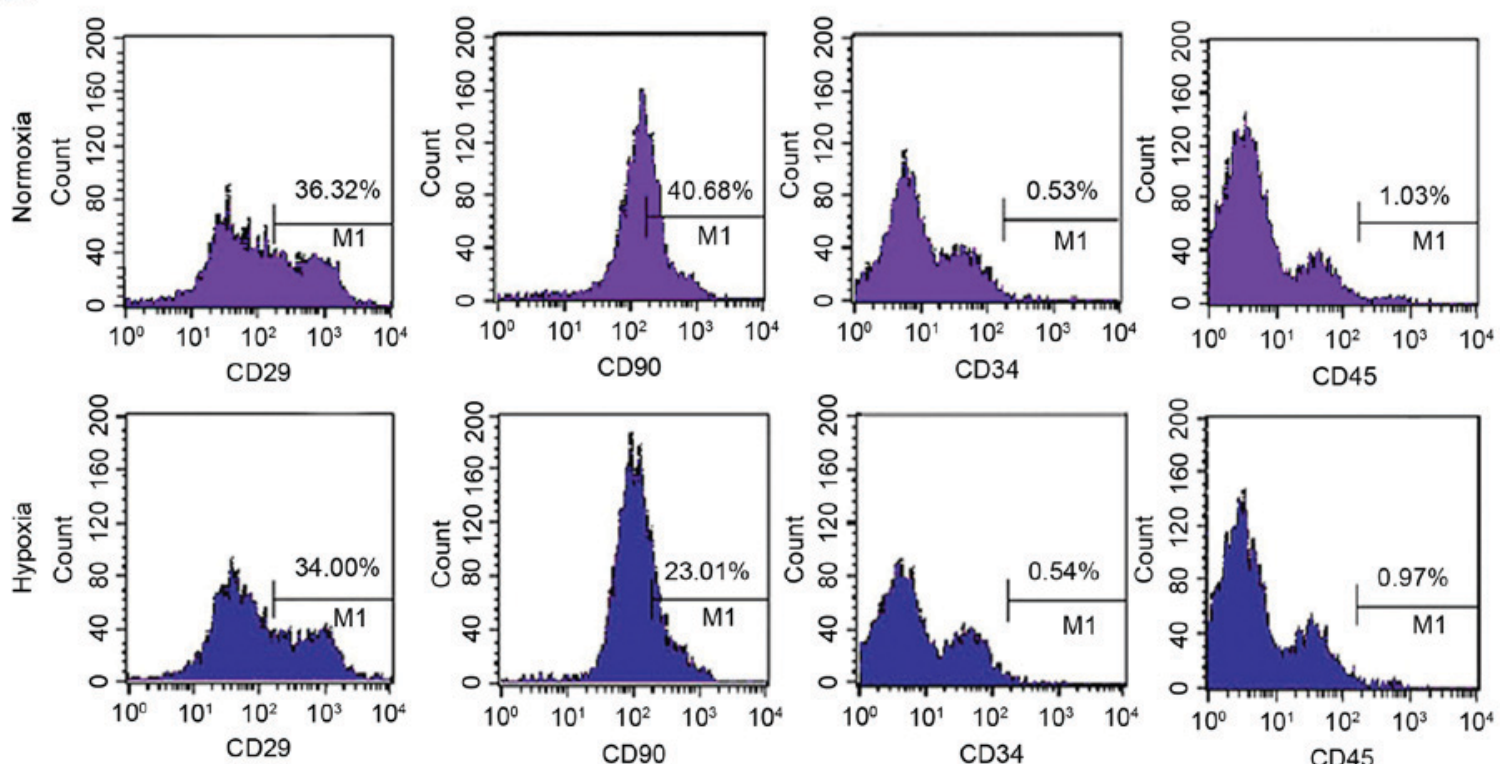

Figure 1. Characteristics of mouse BMDCs cultures. (A) BMDCs were isolated from mouse bone marrow aspirates by adhesion to plates in complete medium By the third passage, a homogeneous population of fibroblast-like cells were obtained in normoxic and hypoxic condition. (B) Immunophenotyping of hMSCs and nMSCs with hematopoietic and mesenchymal markers identified with flow cytometry. BMDC, bone marrow derived cell; CD, cluster of differentiation; h, hypoxia cultured; MSC, mesenchymal stem cell; n, normoxia cultured. 

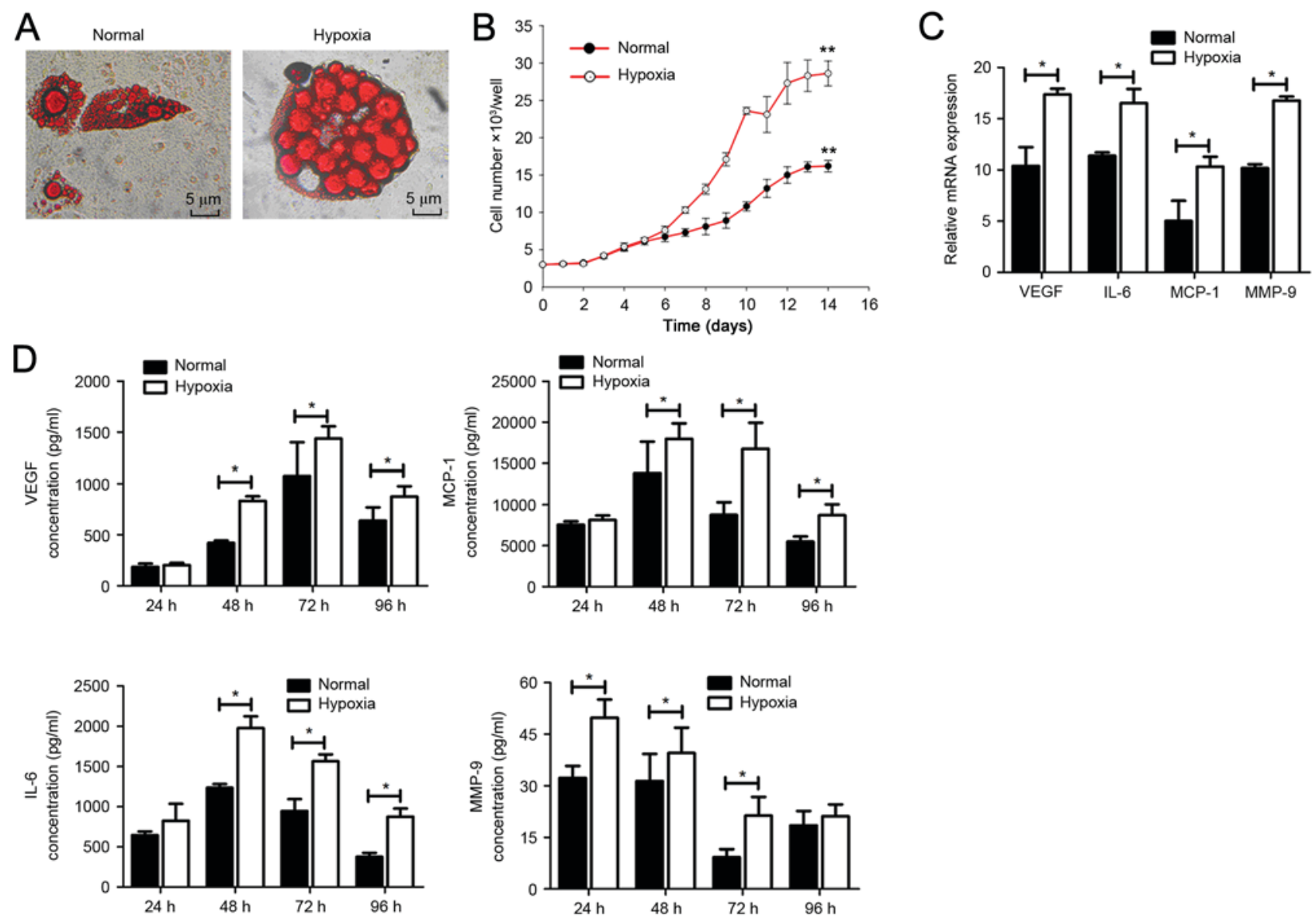

Figure 2. MSCs secrete more growth-promoting related cytokines in hypoxic condition. (A) Adipogenesis was examined by oil red-O staining of MSCs cultured in hypoxic and normoxic environments. (B) Hypoxia induces proliferation increase in MSCs compared with normoxia cultured MSCs, as demonstrated by MTT assay ( $\left.{ }^{* *} \mathrm{P}<0.01\right)$; absorbency was detected every day for 15 days. (C) Reverse transcription-quantitative polymerase chain reaction was used to detect the mRNA expression levels of VEGF, IL-6, MCP-1 and MMP-9 after $48 \mathrm{~h}$ hypoxia treatment (" $\mathrm{P}<0.05$ ). (D) MSCs secrete higher concentrations of VEGF, IL-6, MCP-1 and MMP-9 in hypoxic conditions compared with normoxic-treated cells, as detected by ELISA. IL, interleukin; MCP, monocyte chemoattractant protein; MMP, matrix metallopeptidase; MSC, mesenchymal stem cell; MTT, 3-(4,5-dimethylthiazol-2-yl)-2,5-diphenyltetrazolium bromide; VEGF, vascular endothelial growth factor ( $\mathrm{P}<0.05)$.

group following $48 \mathrm{~h}$ hypoxia treatment $(\mathrm{P}<0.01$; Fig. $2 \mathrm{C})$. In addition, $\mathrm{CM}$ was collected and replaced at $24 \mathrm{~h}$ intervals until $96 \mathrm{~h}$ of culture. ELISA revealed that VEGFA, IL-6, MCP-1 and MMP-9 secretion was increased in hMSCs-CM compared with nMSCs-CM; VEGF, MCP-1 and IL-6 demonstrated a secretion peak between 48 and $72 \mathrm{~h}$, whereas the highest secretion of MMP-9 was in the first $24 \mathrm{~h}$. In addition, the variation between the 4 factors was generally increased in the 24 to $96 \mathrm{~h}$ period under hypoxia induction compared with normoxia condition (Fig. 2D). These results suggested that hypoxic conditions may increase the secretion of growth-related cytokines from MSCs to support graft vascularization and to protect islet cells from apoptosis to avoid islet function loss in the initial period of transplantation.

hMSCs-CM protects islets from hypoxia-induced apoptosis. For the induction of islet apoptosis, islets were cultured in a low-oxygen environment. Subsequently, nMSC-CM or hMSC-CM was introduced to aid islet survival rate in the hypoxic condition. Microscopic evaluations of apoptosis were carried out on the islets stained with FDA/PI; dead cells are stained red and viable cells stained green (Fig. 3A). After $48 \mathrm{~h}$ of culture, apoptosis rates were significantly increased in hypoxia-treated islets $(77.3 \pm 5.7 \%)$ compared with Control islets grown in normoxic conditions $(4.8 \pm 2.1 \%$; $\mathrm{P}<0.05)$; hMSC-CM co-treatment significantly decreased the hypoxia-induced apoptotic rates compared with the nMSCs-CM-treated group $(20.8 \pm 14.2 \%$ and $44.6 \pm 9.8 \%$, respectively; $\mathrm{P}<0.05)$. Apoptotic rates were also analyzed using flow cytometry with PI and Annexin V-FITC labeling. All early and late apoptosis and necrotic cells were identified as the non-survival group. Only Annexin V-FITC)-/PI-cells were identified as the survival group. The survival rate of islets in hypoxic conditions was significantly reduced compared with those cultured in normoxic conditions $(42.3 \pm 6.1$ and $93.8 \pm 2.1 \%$, respectively; $\mathrm{P}<0.01$; Fig. 3B). hMSC-CM treatment significantly improved the survival rate of islets in hypoxic conditions compared with the nMSC-CM treated group $(77.3 \pm 3.2$ and $62.4 \pm 3.8 \%$, respectively; $\mathrm{P}<0.01$; Fig. $3 \mathrm{~B})$. In addition, an in vitro glucose-stimulated insulin secretion test was performed to examine the possible effects of inducible MSC-CM on islet secretory function. Under additive glucose stimulation, insulin secretion was increased in islets co-cultured with the nMSC-CM and hMSC-CM groups in medium (11.1 mM) and high $(27 \mathrm{mM})$ glucose stimulation compared with the hypoxia treatment group. HMSC-CM particularly increased 
A

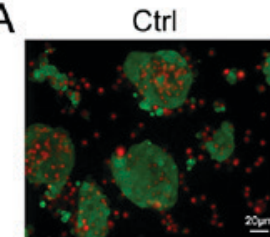

Hypoxia and nMSCS-CM

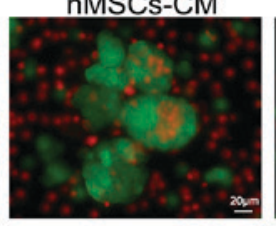

B

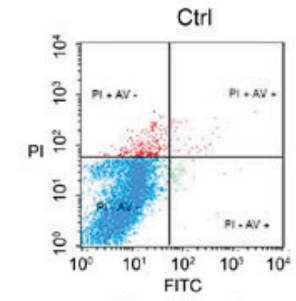

Hypoxia and nMSCS-CM

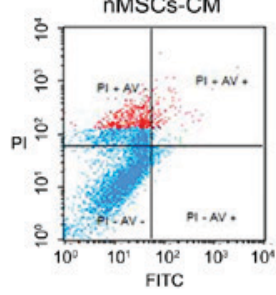

Hypoxia

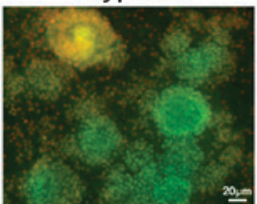

Hypoxia and hMSCs-CM
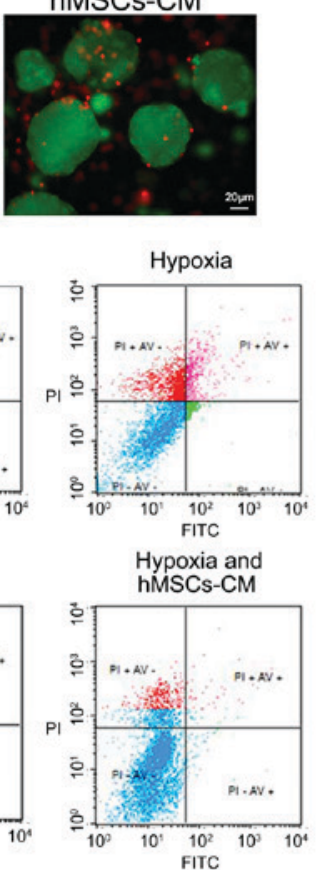

C

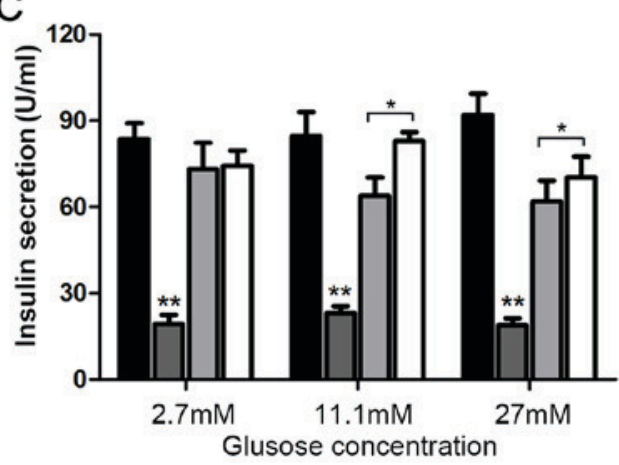

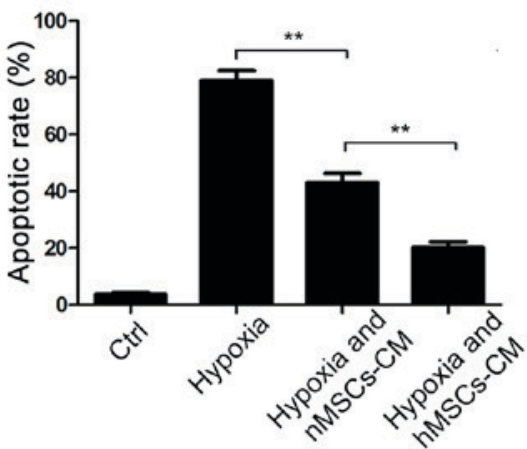

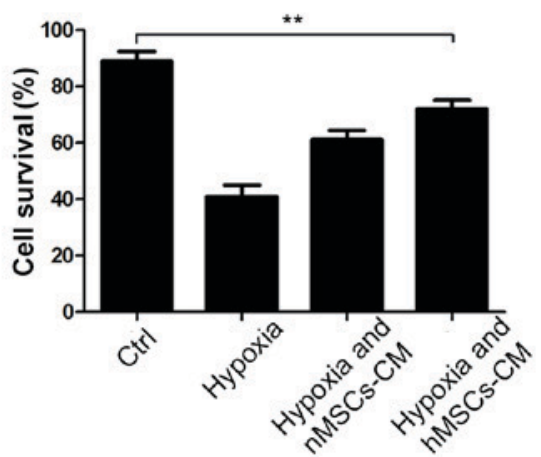

Normal

Hypoxia

Hypoxia and nMSCs-CM

$\square$ Hypoxia and hMSCs-CM

Figure 3. MSC-CM protects islets from apoptosis under hypoxia. (A) FDA/PI test for the islets in hypoxia condition with nMSCs and hMSC CM culture. Scale bar $=20 \mu \mathrm{m}$. Compared with hypoxia mediated group, MSC-CM protected islets from hypoxia induced impairment. hMSCs-CM better prevent islets from apoptosis compared with nMSCs-CM group $\left({ }^{* *} \mathrm{P}<0.01\right)$. (B) Islet cells were cultured with nMSC-CM or hMSC-CM for $48 \mathrm{~h}$ and apoptosis was examined by Annexin V-FITC/PI staining and flow cytometry, Only Annexin V-FITC)-/PI-cells were identified as the survival group ( $\left.{ }^{* * *} \mathrm{P}<0.01\right)$. (C) Islets insulin secretion in different $\mathrm{CM}$ under gradient glucose concentration $(2.7,11.1$ and $27 \mathrm{mM})$ stimulation. CM, conditioned medium; Crtl, control; FITC, fluorescein isothiocyanate; h, hypoxia cultured; MSC, mesenchymal stem cell; $n$, normoxia cultured; PI, propidium iodide $\left({ }^{*} \mathrm{P}<0.05,{ }^{* *} \mathrm{P}<0.01\right)$.

insulin secretion compared with the nMSC-CM group in medium $(11.1 \mathrm{mM})$ and high $(27 \mathrm{mM})$ glucose stimulation $(\mathrm{P}<0.05)$, whereas less difference was detected in the low $(2.7 \mathrm{mM})$ glucose concentration stimulation group between the hMSC-CM and nMSC-CM groups (Fig. 3C).

Co-transplantation of hMSCs improves the function of transplanted islets in vivo. Mice were treated with STZ to induce diabetes. A total of 250-400 islets were transplanted under the kidney capsule, either alone or co-transplanted with nMSCs or hMSCs; blood glucose levels were monitored every 3 days for 30 days. The minimal number of islets required to reverse the impaired glucose tolerance condition to reach normoglycemia was $\sim 400$ in mice in the Islet-only group, $\sim 300$ in the Islet + nMSCs group and $\sim 250$ in the Islets + hMSCs group (Fig. 4).

Co-transplantation of islets with hMSCs reverses high postprandial blood glucose levels in diabetic mice. Cumulative diabetes reversal curves were described in the transplant model following islets transplantation. Post-operative transient hyperglycemia due to fasting and surgery was observed in all groups. At 4 weeks following transplantation, $100 \%$ of mice in the 300 Islets + hMSCs, the 250 Islets + hMSCs and the 300 

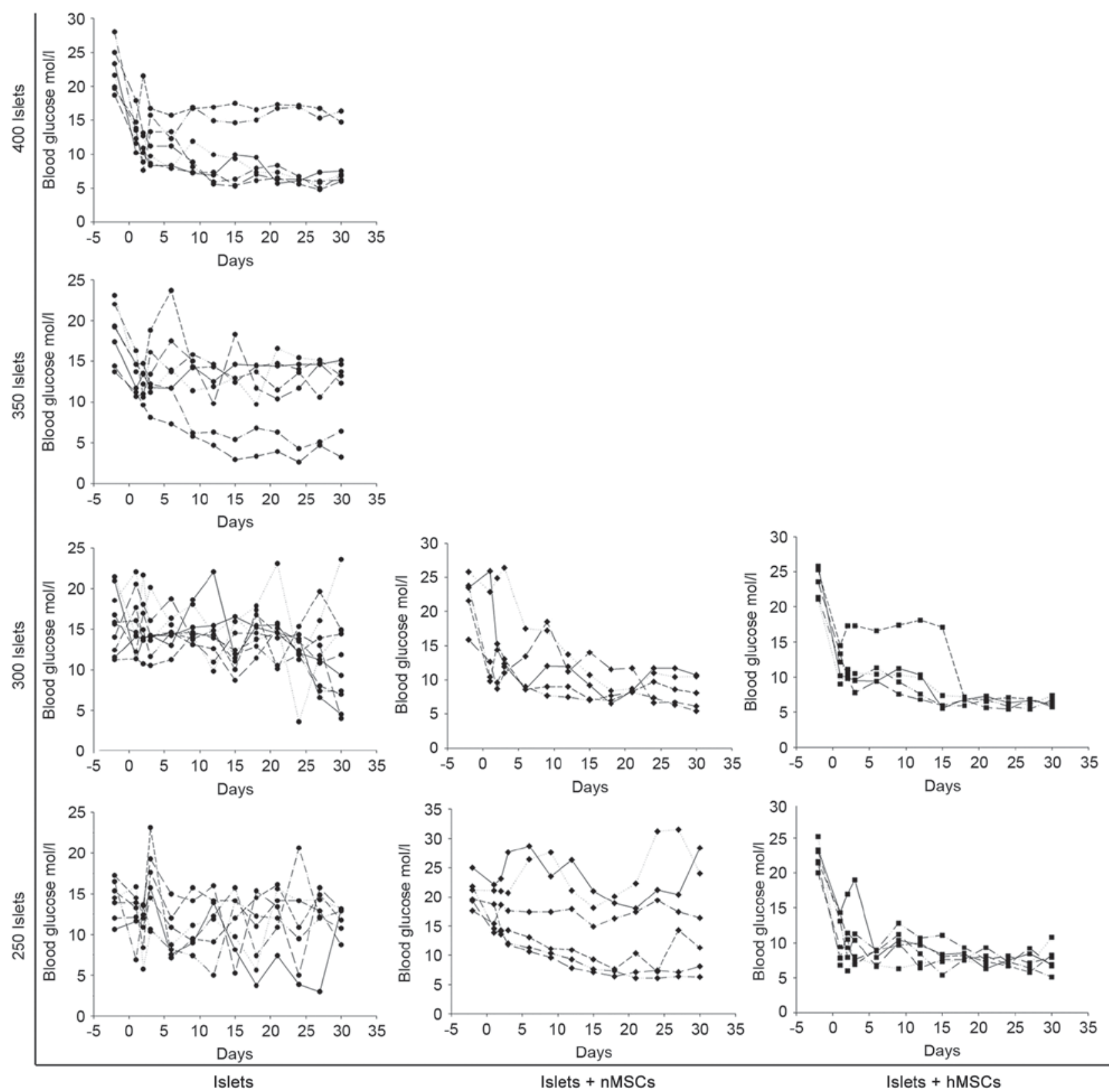

Figure 4. Therapeutic application in the context of glucose uptake with co-transplantation of hMSCs and islets. A total of 250-400 islets were transplanted under the kidney capsule of syngeneic mice, either alone or co-transplanted with nMSCs or hMSCs; blood glucose concentration was measured every 3 days for 30 days. In the Islets + hMSCs co-transplantation group, only 250 islets were used to reverse postprandial high blood-glucose, 300 islets in the Islets + nMSCs group and 400 islets in Islets-alone group were required to reach similar therapeutic effects. H, hypoxia cultured; MSC, mesenchymal stem cell; n, normoxia cultured.

Islets + nMSCs groups were restored to euglycemia compared with $50 \%$ of mice in the 250 Islets + nMSCs, $72 \%$ in the 400 Islets, $28 \%$ in the 350 Islets, $20 \%$ in the 300 Islets and $0 \%$ in 250 Islets groups (Fig. 5A). Mice in the two Islets + hMSCs groups exhibited higher diabetes reversal ratios compared with mice in the Islets-alone, Islets + nMSCs or empty (no islet transplantation) groups. A glucose tolerance test was performed to confirm the effects of Islets + hMSCs transplantation on STZ-induced diabetic mice at 30 days following islet implantation in 5 age-matched pairs of BALB/c mice. The normal control group was mice without STZ treatment and the empty group was STZ induced diabetes mice without any graft transplantation. hMSCs co-transplantation significantly accelerated glycemic utilization following glucose intake compared with MSCs and islets only group $(\mathrm{P}<0.05$; Fig. $5 \mathrm{~B}$ and $\mathrm{C})$.
mRNAmicroarraydatainislets transplantedmice. Hierarchical clustering analysis of microarray data revealed mouse serum mRNA expression of Islets + hMSCs, Islets + nMSCs and the Islets-only control groups ( $\mathrm{n}=3 \mathrm{mice} / \mathrm{group})$. Abnormally increased cytokines were detected between Islets + hMSCs group and Islets + nMSCs and control groups (unaltered mRNAs not shown; Fig. 6).

\section{Discussion}

Incomplete graft revascularization and hypoxic conditions impede the clinical use of islet transplantation (28-30). Early hypoxia-related islet death following intramuscular transplantation is the major factor for loss of islets and results in an increased rate of transplantation failure $(31,32)$. In previous 

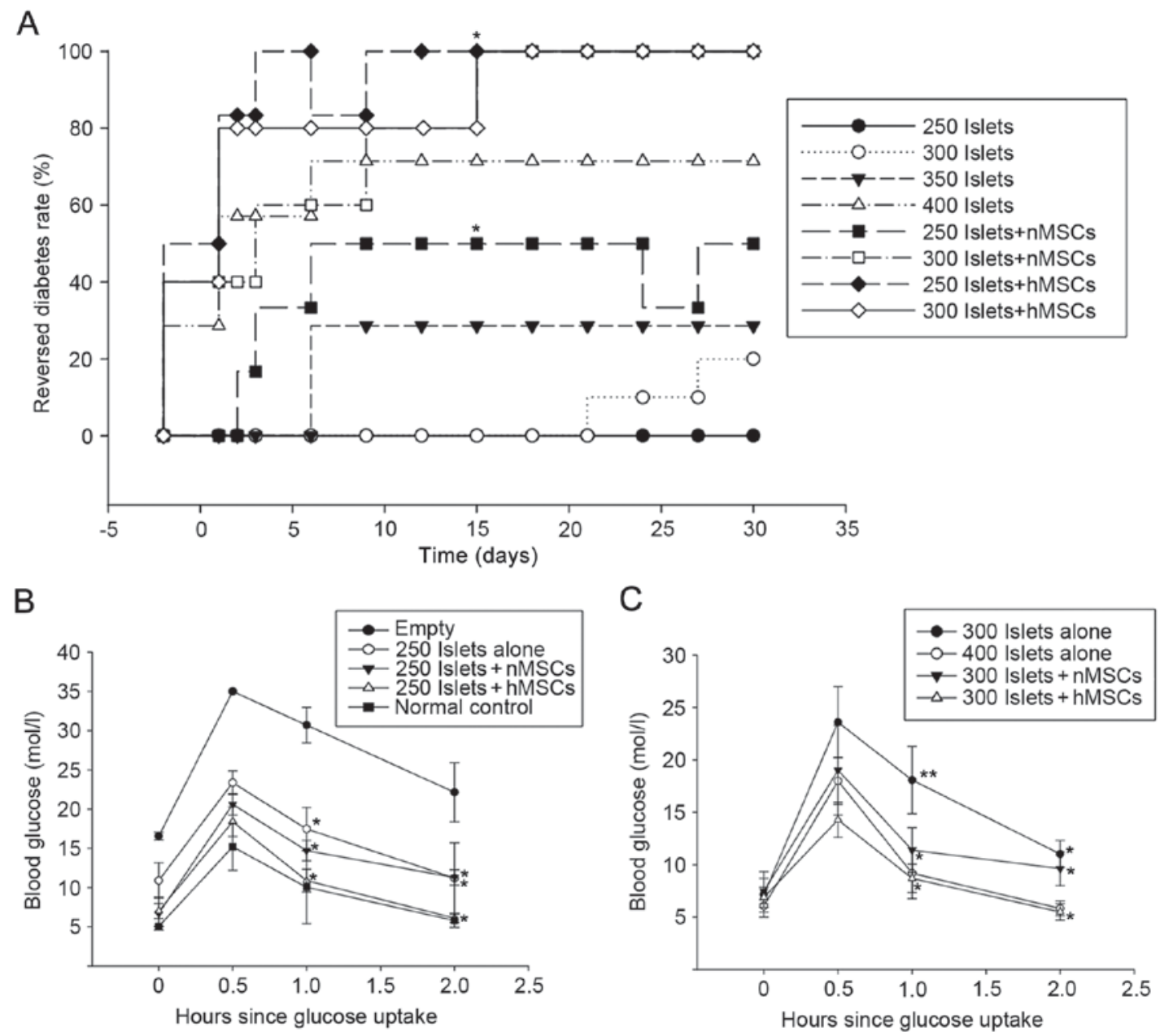

Figure 5. Reduction in minimal islet mass required to reverse diabetes by co-transplantation with hMSCs. (A) Cumulative diabetes reversal curves (recipients reversed rate) demonstrated that islet graft function was improved with MSCs co-transplantation, particularly co-transplantation with hMSCs ("P<0.05), (B and C) Co-transplantation of hMSCs improves islet graft function. Intravenous glucose-tolerance test was performed on day 30 in all transplant recipients. A total of 5 age-matched normal BALB/c mice or diabetic mice without islets transplantation were used as a control. The normal control group constituted mice without STZ treatment and the empty group was STZ induced diabetes mice without any graft transplantation. Glucose utilization was faster in Islets + hMSCs recipients compared with mice receiving islets alone and Islets + nMSCs group $\left({ }^{* *} \mathrm{P}<0.01,{ }^{*} \mathrm{P}<0.05\right)$ in both 250 and 300 islets transplanted group. $\mathrm{H}$, hypoxia cultured; MSCs, mesenchymal stem cells; n, normoxia cultured; STZ, streptozotocin.

studies, E26 avian leukemia oncogene 1, solute carrier family 30 member 8 and zinc transporter have been reported to improve the survival rate of islets in hypoxic conditions $(33,34)$; however, maintenance of islet function remains unsatisfactory.

A previous study reported that co-transplantation of islets with MSCs was associated with enhanced islet graft vascularization and functional recovery (35). In another previous study, a number of beneficial effects on blood glucose regulation were observed in islets + nMSC transplanted mice (8). However, these observations did not support the hypothesis that co-transplantation of islets with MSCs is sufficient for a complete reversal of hyperglycemia in diabetic mice. The present study pretreated MSCs in hypoxic conditions prior to local kidney capsule transplantation in diabetic mice, to evaluate their effects on islets and islet function. Hypoxia has a strong effect on several aspects of cell biology, including metabolism, angiogenesis, innate immunity and stemness induction (36). In MSCs, stem cells pre-cultured in hypoxic conditions could improve the potential of their tissue regenerative function (19). Similarly, hypoxic conditions induced MSCs pro-angiogenic and growth-related genes expression and activated certain major receptors for hepatocyte growth factor (HGF) and enhanced cMet signaling to improve their tissue regenerative potential $(19,37)$. Therefore, the present study hypothesized that hMSCs may also secrete increased levels of cytokines including VEGFA, IL-6, MCP-1 and MMP-9 to support graft vascularization, certain pro-angiogenic and growth rate of MSCs.

In a previous study, VEGFA, known as a vital angiogenesis-related factor in islets transplantation, was found to improve intra-islet vascular reformation to protect islet survival (38). IL-6 is a pleiotropic cytokine with complex roles in inflammation and metabolism. During islets transplantation, a previous study demonstrated that IL-6 robustly activated signal transducer and activator of transcription 3 , which is involved in autophagy, and as a result directly protected islet cells from apoptosis by stimulation of autophagy (39). Several previous studies have revealed that MCP-1 and MMP-9 possess various potential clinical implications in islet transplantation. For example, one study demonstrated that recipient C-C motif chemokine 2/MCP-1 may be a major pharmacological target for the control of potentially islet damaging reactions at the site of transplant (40). MMPs are proteolytic enzymes that are involved 


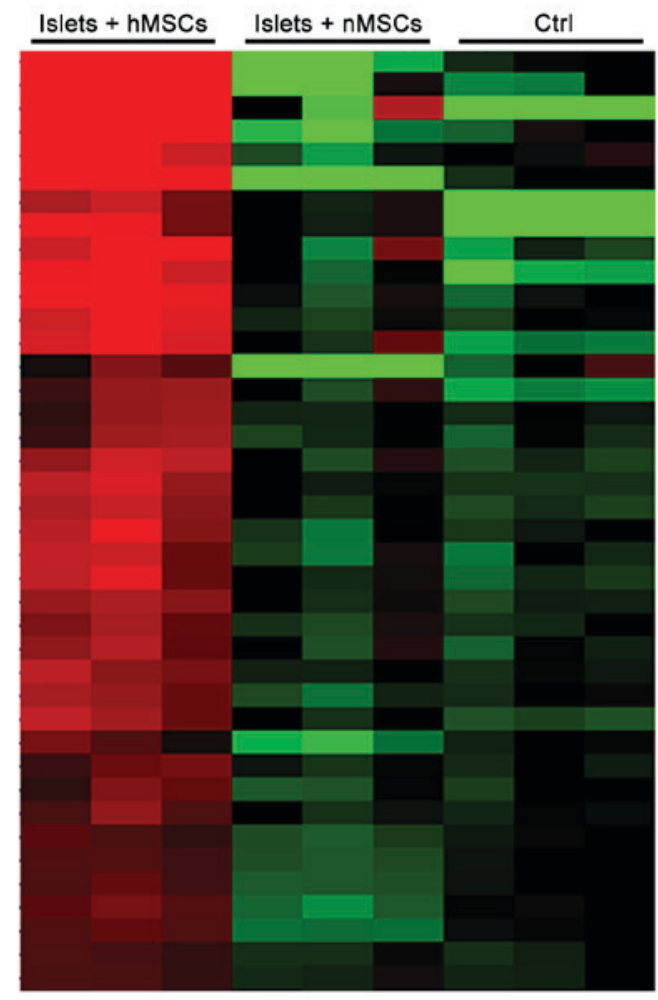

Gene (mouse)
Chi3l3
Fermt3
Tuba4a
Chi313
Ptprc
Fos
Coro1a
Btg1
Ccl5
Ctss
Sumo2
Tpm2
Gzma
Cd151
Lgals1
Tyrobp
Cd52
Ly6c1
Sirpb1
Mpeg1
Ms4a4b
Cd53
Hspa8
Ms4a6b
Pgk1
Gimap4
Psap
Fam49b
Mcl1
Actr3
S100a4
Tesc
Lgals3
Pla2g7
Ugt1a1
Clec7a
Fpr2
II1b
Ap1s2
Sac3d1

Figure 6. Variation of gene expression profile in mouse serum following islet + hMSCs co-transplantation. Hierarchical clustering analysis demonstrated the most increased ( $>3$ times) coding mRNA mouse gene in the Islets + hMSCs group compared with the Islets + nMSC and control groups. Serum was collected 10 days following transplantation surgery and each group contained three genomically matched mice. $\mathrm{H}$, hypoxia cultured; MSC, mesenchymal stem cell; n, normoxic cultured.

in the breakdown of extracellular matrix proteins. Inhibition of MMP-9 was reported to results in increased amyloid deposition and apoptosis in mouse islets, which suggested that MMP-9 may serve a physiological role in limiting islet amyloid deposition and protects islets from amyloid-induced toxicity (41). As a result, high secretion of these cytokines in hMSCs may increase the concentration of these protective factors around transplanted islet cells. In this condition, a prospective environment suitable for improved islet survival is created, which decreases the number of islets required to reach the desired blood glucose concentration. This method avoids the use of artificial gene delivery techniques to increase the secretion of cytokines of transplanted cells in mouse experiments and the risk of transfection toxicity $(29,32)$. Transplantation is preferable to the danger of biological transfection cytotoxicity.

The present study demonstrated that hMSC-CM was able to effectively protect the islets from apoptotic death and increased the insulin secretion of the islet compared with nMSCs. A notable reduction was observed in the minimal mass of islets required to reverse diabetes in mice. In this respect, islet function parameters were superior in the 250 Islets + hMSCs group compared with the other treatment groups and was sufficient to control the blood glucose concentration; previously, islets were required from 2 to 4 donors to treat each recipient in order to achieve a state of complete insulin independence (4). Clinical islet transplantation should proceed towards the use of single donor organs instead of multiple donors. Therefore, any improvements leading to the reduction of the number of islets required in transplanted islets may be clinically important. Insulin independence following islet transplantation from a single donor and decreasing procedural complications attributed to multiple islet infusions are of great importance.

In conclusion, results from the present study demonstrated that the underlying mechanisms modulating pancreatic islet viability may be attributable to the paracrine mediators VEGFA, IL-6, MCP-1 and MMP-9 secreted by hMSCs; co-transplantation with hMSCs may reduce the minimal islet mass required to reverse diabetes in mice. In addition, a number of concerns about the use of hMSC may be investigated in the context of allogeneic islet transplantation for future proper glyco-metabolic control.

\section{Acknowledgments}

The authors would like to thank Dr Rashid. A. Tabassum (The Second Affiliated Hospital of Zhejiang University, Hangzhou, China) who provided language and writing guidance.

\section{Funding}

The present study was supported by The Scientific Foundation of Zhejiang Province (grant no. LY14H160033).

\section{Availability of data and materials}

The datasets used and/or analyzed during the current study are available from the corresponding author on reasonable request.

\section{Authors' contributions}

QPX made substantial contributions to the concept and design of the present study. CX performed the experiments, wrote the paper and reviewed and edited the manuscript. Both authors read and approved the final manuscript.

\section{Ethics approval and consent to participate}

All procedures performed on animals were in accordance with ethical standards and were approved by the ethics committee of The Second Affiliated Hospital of Zhejiang University (Hangzhou, China).

\section{Patient consent for publication}

Not applicable.

\section{Competing interests}

The authors declare that they have no competing interests.

\section{References}

1. Abdel-Moneim A, Bakery HH and Allam G: The potential pathogenic role of IL-17/Th17 cells in both type 1 and type 2 diabetes mellitus. Biomed Pharmacother 101: 287-292, 2018. 
2. Koro CE, Bowlin SJ, Bourgeois N and Fedder DO: Glycemic control from 1988 to 2000 among U.S. adults diagnosed with type 2 diabetes: A preliminary report. Diabetes care 27: 17-20, 2004.

3. El-Badri N and Ghoneim MA: Mesenchymal stem cell therapy in diabetes mellitus: Progress and challenges. J Nucleic Acids 2013: 194858, 2013.

4. Shapiro AM, Lakey JR, Ryan EA, Korbutt GS, Toth E, Warnock GL, Kneteman NM and Rajotte RV: Islet transplantation in seven patients with type 1 diabetes mellitus using a glucocorticoid-free immunosuppressive regimen. N Engl J Med 343: 230-238, 2000.

5. Barton FB, Rickels MR, Alejandro R, Hering BJ, Wease S, Naziruddin B, Oberholzer J, Odorico JS, Garfinkel MR, Levy M, et al: Improvement in outcomes of clinical islet transplantation: 1999-2010. Diabetes Care 35: 1436-1445, 2012.

6. Ricordi C: Islet transplantation: A brave new world. Diabetes 52: 1595-1603, 2003

7. Brandhorst H, Asif S, Andersson K, Mönch J, Friedrich O, Rämsch-Günther N, Rämsch C, Steffens M, Lambrecht J, Schräder T, et al: The effect of truncated collagenase class I isomers on human islet isolation outcome. Transplantation 90: 334-335, 2010

8. Ito T, Itakura S, Todorov I, Rawson J, Asari S, Shintaku J, Nair I, Ferreri K, Kandeel F and Mullen Y: Mesenchymal stem cell and islet co-transplantation promotes graft revascularization and function. Transplantation 89: 1438-1445, 2010.

9. Rackham CL, Chagastelles PC, Nardi NB, Hauge-Evans AC, Jones PM and King AJ: Co-transplantation of mesenchymal stem cells maintains islet organisation and morphology in mice. Diabetologia 54: 1127-1135, 2011.

10. Perez-Basterrechea M, Obaya AJ, Meana A, Otero J and Esteban MM: Cooperation by fibroblasts and bone marrow-mesenchymal stem cells to improve pancreatic rat-to-mouse islet xenotransplantation. PLoS One 8: e73526, 2013.

11. Berman DM, Willman MA, Han D, Kleiner G, Kenyon NM, Cabrera O, Karl JA, Wiseman RW, O'Connor DH, Bartholomew AM and Kenyon NS: Mesenchymal stem cells enhance allogeneic islet engraftment in nonhuman primates. Diabetes 59: 2558-2568, 2010.

12. Sakata N, Goto M, Yoshimatsu G, Egawa S and Unno M: Utility of co-transplanting mesenchymal stem cells in islet transplantation. World J Gastroenterol 17: 5150-5155, 2011.

13. Sordi V, Melzi R, Mercalli A, Formicola R, Doglioni C, Tiboni F, Ferrari G, Nano R, Chwalek K, Lammert E, et al: Mesenchymal cells appearing in pancreatic tissue culture are bone marrow-derived stem cells with the capacity to improve transplanted islet function. Stem Cells 28: 140-151, 2010.

14. Golocheikine A, Tiriveedhi V, Angaswamy N, Benshoff N, Sabarinathan R and Mohanakumar T: Cooperative signaling for angiogenesis and neovascularization by VEGF and HGF following islet transplantation. Transplantation 90: 725-731, 2010

15. Ding Y, Xu D, Feng G, Bushell A, Muschel RJ and Wood KJ: Mesenchymal stem cells prevent the rejection of fully allogenic islet grafts by the immunosuppressive activity of matrix metalloproteinase-2 and -9. Diabetes 58: 1797-1806, 2009.

16. Karaoz E, Genc ZS, Demircan PC, Aksoy A and Duruksu G: Protection of rat pancreatic islet function and viability by coculture with rat bone marrow-derived mesenchymal stem cells. Cell Death Dis 1: e36, 2010.

17. Xie QP, Huang H, Xu B, Dong X, Gao SL, Zhang B and Wu YL: Human bone marrow mesenchymal stem cells differentiate into insulin-producing cells upon microenvironmental manipulation in vitro. Differentiation 77: 483-491, 2009.

18. Grayson WL, Zhao F, Izadpanah R, Bunnell B and Ma T: Effects of hypoxia on human mesenchymal stem cell expansion and plasticity in 3D constructs. J Cell Physiol 207: 331-339, 2006

19. Rosova I, Dao M, Capoccia B, Link D and Nolta JA: Hypoxic preconditioning results in increased motility and improved therapeutic potential of human mesenchymal stem cells. Stem Cells 26: 2173-2182, 2008

20. Hung SC, Pochampally RR, Chen SC, Hsu SC and Prockop DJ: Angiogenic effects of human multipotent stromal cell conditioned medium activate the PI3K-Akt pathway in hypoxic endothelia cells to inhibit apoptosis, increase survival and stimulate angiogenesis. Stem Cells 25: 2363-2370, 2007.

21. Dao MA, Pepper KA and Nolta JA: Long-term cytokine production from engineered primary human stromal cells influences human hematopoiesis in an in vivo xenograft model. Stem cells 15: 443-454, 1997
22. D'Amato F, Noli B, Brancia C, Cocco C, Flore G, Collu M, Nicolussi P and Ferri GL: Differential distribution of VGF-derived peptides in the adrenal medulla and evidence for their selective modulation. J Endocrinol 197: 359-369, 2008.

23. Livak KJ and Schmittgen TD: Analysis of relative gene expression data using real-time quantitative PCR and the 2(-Delta Delta C(T)) method. Methods 25: 402-408, 2001.

24. Ichii H, Wang X, Messinger S, Alvarez A, Fraker C, Khan A, Kuroda Y, Inverardi L, Goss JA, Alejandro R and Ricordi C: Improved human islet isolation using nicotinamide. Am J Transplant 6: 2060-2068, 2006.

25. Pittenger MF, Mackay AM, Beck SC, Jaiswal RK, Douglas R, Mosca JD, Moorman MA, Simonetti DW, Craig S and Marshak DR: Multilineage potential of adult human mesenchymal stem cells. Science 284: 143-147, 1999.

26. Dominici M, Le Blanc K, Mueller I, Slaper-Cortenbach I, Marini F, Krause D, Deans R, Keating A, Prockop Dj and Horwitz E: Minimal criteria for defining multipotent mesenchymal stromal cells. The international society for cellular therapy position statement. Cytotherapy 8: 315-317, 2006.

27. Luo JZ, Xiong F, Al-Homsi AS, Roy T and Luo LG: Human BM stem cells initiate angiogenesis in human islets in vitro. Bone Marrow Transplantat 46: 1128-1137, 2011.

28. Hajizadeh-Saffar E, Tahamtani Y, Aghdami N, Azadmanesh K, Habibi-Anbouhi M, Heremans Y, De Leu N, Heimberg H, Ravassard P, Shokrgozar MA and Baharvand H: Inducible VEGF expression by human embryonic stem cell-derived mesenchymal stromal cells reduces the minimal islet mass required to reverse diabetes. Sci Rep 5: 9322, 2015.

29. Sato $Y$, Endo H, Okuyama H, Takeda T, Iwahashi H, Imagawa A, Yamagata K, Shimomura I and Inoue M: Cellular hypoxia of pancreatic beta-cells due to high levels of oxygen consumption for insulin secretion in vitro. J Biol Chem 286: 12524-12532, 2011.

30. Buchwald P: A local glucose-and oxygen concentration-based insulin secretion model for pancreatic islets. Theor Biol Med Model 8: 20, 2011.

31. Espes D, Lau J, Quach M, Banerjee U, Palmer AF and Carlsson PO: Cotransplantation of Polymerized Hemoglobin Reduces $\beta$-Cell Hypoxia and Improves $\beta$-Cell Function in Intramuscular Islet Grafts. Transplantation 99: 2077-2082, 2015.

32. Liljeback H, Grapensparr L, Olerud J and Carlsson PO: Extensive loss of islet mass beyond the first day after intraportal human islet transplantation in a mouse model. Cell Transplant 25: 481-489, 2016.

33. Qiao N, Xu C, Zhu YX, Cao Y, Liu DC and Han X: Ets-1 as an early response gene against hypoxia-induced apoptosis in pancreatic $\beta$-cells. Cell Death Dis 6: e1650, 2015.

34. Gerber PA, Bellomo EA, Hodson DJ, Meur G, Solomou A, Mitchell RK, Hollinshead M, Chimienti F, Bosco D, Hughes SJ, et al: Hypoxia lowers SLC30A8/ZnT8 expression and free cytosolic Zn2+ in pancreatic beta cells. Diabetologia 57: 1635-1644, 2014.

35. Sakata N, Chan NK, Chrisler J, Obenaus A and Hathout E: Bone marrow cell cotransplantation with islets improves their vascularization and function. Transplantation 89: 686-693, 2010.

36. Majmundar AJ, Wong WJ and Simon MC: Hypoxia-inducible factors and the response to hypoxic stress. Mol Cell 40: 294-309, 2010.

37. Lampert FM, Kutscher C, Stark GB and Finkenzeller G: Overexpression of Hif- $1 \alpha$ in mesenchymal stem cells affects cell-autonomous angiogenic and osteogenic parameters. J Cell Biochem 117: 760-768, 2016.

38. Mao D, Zhu M, Zhang X, Ma R, Yang X, Ke T, Wang L, Li Z, Kong D and Li C: A macroporous heparin-releasing silk fibroin scaffold improves islet transplantation outcome by promoting islet revascularisation and survival. Acta Biomater 59: 210-220, 2017.

39. Linnemann AK, Blumer J, Marasco MR, Battiola TJ, Umhoefer HM, Han JY, Lamming DW and Davis DB: Interleukin 6 protects pancreatic beta cells from apoptosis by stimulation of autophagy. FASEB J 31: 4140-4152, 2017.

40. Melzi R, Mercalli A, Sordi V, Cantarelli E, Nano R, Maffi P, Sitia G, Guidotti LG, Secchi A, Bonifacio E and Piemonti L: Role of CCL2/MCP-1 in islet transplantation. Cell Transplant 19: 1031-1046, 2010.

41. Meier DT, Tu LH, Zraika S, Hogan MF, Templin AT, Hull RL, Raleigh DP and Kahn SE: Matrix metalloproteinase-9 protects islets from amyloid-induced toxicity. J Biol Chem 290: 30475-30485, 2015.

This work is licensed under a Creative Commons Attribution-NonCommercial-NoDerivatives 4.0 International (CC BY-NC-ND 4.0) License. 